\title{
Ecoulement d'un matériau granulaire à travers un orifice : effet de paroi
}

\author{
Flow of a granular media out of a silo \\ through an orifice: effect of walls
}

\author{
N.E. ABRIAK \\ Chargé de Recherches, Responsable du Laboratoire de Mécanique \\ des sols, Ecole des Mines de Douai ${ }^{*}$ \\ R. GOURVES \\ Maître de conférence au CUST de Clermont-Ferrand ${ }^{\circ}$
}

Rev. Franç. Géotech. n 61, pp. 47-61 (décembre 1992)

\section{Résumé}

On présente l'influence de la nature de la paroi sur le comportement statique et cinématique du matériau analogique de SCHNEEBELI. Cette étude a été menée sur un silo bidimensionnel à parois lisses ou rugueuses, à fond plat ou à trémie.

Une étude statique consistant à déterminer la distribution des efforts dans un silo chargé a aussi été effectuée. Les résultats expérimentaux s'accordent assez bien avec la théorie de JANSSEN. Cette étude montre aussi que certains phénomènes propres aux écoulements des milieux granulaires s'expliquent qualitativement et quantitativement par la seule considération des déplacements des particules. Par ailleurs, ces écoulements ont été classés en deux modes suivant la rugosité et l'inclinaison de la trémie.

\footnotetext{
Abstract

The effect of the walls on the statical and kinematical behaviour of an analogical material (or SCHNEEBELI's one) is presented. A two dimensional model with smooth or wrinkled walls, plane bottom or with a hopper, has been used during this study.

The stress distribution within the material in the silo is determined by means of a static analysis ; this study confirms the results of JANSSEN's theory, improving the experimental method. Some phenomena particular to flow of granular media are explained, in a qualitative and quantitative manner, by means of rolls displacements.
} 


\section{INTRODUCTION}

Plusieurs laboratoires de recherches se sont penchés sur les problèmes des effets de parois au travers d'expériences reproduisant partiellement les conditions industrielles. Certains auteurs $(2,6,10)$ ont tenté d'analyser les différentes situations en créant des modèles approchant le plus possible la réalité en vue d'élaborer des théories de plus en plus fines.

La plupart des recherches ultérieures ont porté sur la détermination expérimentale de la charge totale exercée sur le fond des silos $(3,9)$. A partir de ces résultats, les auteurs déduisent la charge supportée par la paroi puis, par dérivation, la contrainte exercée sur cette dernière. De nombreuses études ont montré que la distribution des efforts varie énormément qu'il s'agisse de matériaux compressibles ou de matériaux dilatants $(8,9)$.

\section{CALCUL DES EFFORTS AU SEIN DE LA COLONNE D'ESSAI}

\subsection{Méthode expérimentale (GOURVES et FAUGERAS)}

Pour étudier l'état des contraintes au sein d'un matériau analogique enfermé entre deux parois rigides verticales très rapprochées, on a utilisé la méthode de GOURVES et FAUGERAS qui est basée sur le principe suivant : plusieurs rouleaux sont déplacés suivant leur axe longitudinal ; leur résistance à l'enfoncement étant proportionnelle aux forces radiales les sollicitant, la connaissance de la force nécessaire pour les déplacer permet de calculer l'état de contrainte par une simple relation.

Cette force peut être mise sous la forme suivante:

$$
F_{z}=\lambda \sigma_{z}+\mu \sigma_{x} \text { et } F_{x}=\lambda \sigma_{x}+\mu \sigma_{z}
$$

$\lambda$ et $\mu$ sont des coefficients qui dépendent des caractéristiques géométriques de la plaque utilisée pour déplacer les rouleaux. Pour déterminer ces coefficients, il est nécessaire d'opérer dans un champ de contrainte connu. Pour ce faire, GOURVES et FAUGERAS (5) ont conçu une machine de compression biaxiale. Ainsi, les contraintes macroscopiques à l'intérieur du massif sont déterminées à partir des relations suivantes :

$$
\begin{aligned}
& \sigma_{v}=\left[1 /\left(\lambda^{2}-\mu^{2}\right)\right]\left(\lambda F_{z}-\mu F_{x}\right) \\
& \sigma_{h}=\left[1 /\left(\lambda^{2}-\mu^{2}\right)\right]\left(\lambda F_{x}-\mu F_{z}\right)
\end{aligned}
$$

On a choisi, pour ce travail, de ne mesurer que la contrainte moyenne $\mathrm{P}=\left[\left(\sigma_{v}+\sigma_{h}\right) / 2\right]$ en utilisant une tige de section circulaire de diamètre $2 \mathrm{~cm}$.

\subsection{Méthode de JANSSEN}

JANSSEN a étudié l'équilibre d'une couche horizontale de matière ensilée d'épaisseur $\mathrm{dZ}$ soumise à des charges verticales et horizontales (fig. 1).

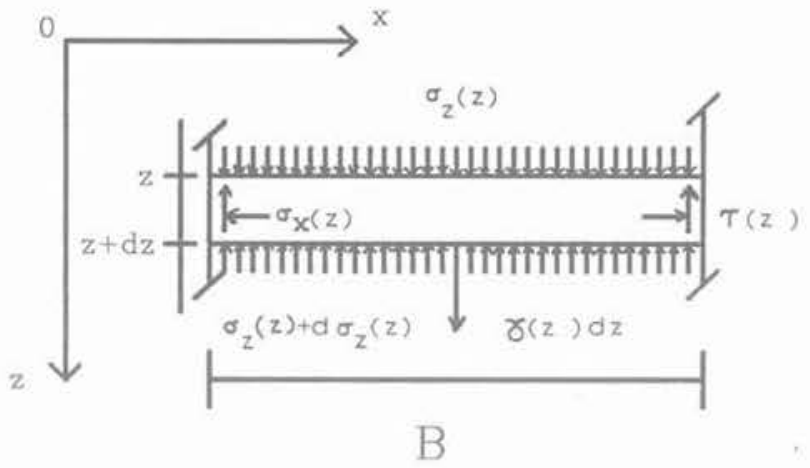

Fig. 1. - Représentation d'une tranche et des efforts qui s'y exercent.

Fig. 1. - Representation of slice and of force acting on it.

Si on considère le bilan des forces dans la direction $\mathrm{Z}$

$$
S \sigma_{z}+\gamma \mathrm{SdZ}-\tau \mathrm{PdZ}-\left(\sigma_{z}+\left(\mathrm{d} \sigma_{z}\right) \mathrm{s}=0\right.
$$

d'où :

$$
\left(\mathrm{d} \sigma_{z} / \mathrm{dZ}\right)+\left[(\mathrm{ktg} \phi / \mathrm{Rh}) \sigma_{2}\right]=\gamma
$$

$\sigma_{2}$ : contrainte verticale sur une section située à la côte Z,

$\sigma_{\mathrm{x}}$ : contrainte horizontale sur une section située à la côte Z,

$\gamma$ : poids volumique de la couche à la profondeur Z,

$\varphi$ : angle de frottement interne,

$\phi$ : angle de frottement paroi/grain,

$\tau$ : contrainte de cisaillement,

$\mathrm{Rh}$ : rayon hydraulique dans le cas du modèle bidimensionnel $\mathrm{Rh}=\mathrm{B} / 2$,

$\mathrm{B}$ : diamètre de la colonne,

$P$ : périmètre.

La solution de l'équation (1) est de la forme:

$$
\begin{aligned}
& \sigma_{z}=(\gamma \mathrm{Rh} / \mathrm{Ktg} \phi)[1-\exp [(-\mathrm{K} \operatorname{tg} \phi / \mathrm{Rh}) \mathrm{Z}]\} \\
& \sigma_{x}=K \sigma_{z} ; \mathrm{K}=[(1-\sin \varphi) /(1+\sin \varphi)]
\end{aligned}
$$

Dans le cas où la surface libre du massif est chargée, la solution de (1) est de la forme:

$$
\begin{aligned}
\sigma_{2}(\mathrm{Z}) & =A_{0} \exp [(\mathrm{K} \operatorname{tg} \phi / \mathrm{Rh}) \mathrm{Z}] \\
& +(\gamma \mathrm{Rh} / \mathrm{K} \operatorname{tg} \phi)\{1-\exp [(-\mathrm{K} \operatorname{tg} \phi / \mathrm{Rh}) \mathrm{Z}]\}
\end{aligned}
$$

$\mathrm{Q}$ : charge totale.

$\mathrm{A}_{0}$ se détermine par passage à la limite ; pour $\mathrm{Z}=0$ nous avons :

$$
\left.\sigma_{2}(0)=A_{0} \text { donc } A_{0}=Q / S\right) ;
$$

d'où :

$$
\begin{aligned}
\sigma_{\mathrm{z}}(\mathrm{Z}) & =(\mathrm{Q} / \mathrm{S}) \exp [(-\mathrm{K} \operatorname{tg} \phi / \mathrm{Rh}) \mathrm{Z}] \\
& +(\gamma \mathrm{Rh} / \mathrm{Ktg} \phi)[1-\exp [(-\mathrm{K} \operatorname{tg} \phi / \mathrm{Rh}) \mathrm{Z}]
\end{aligned}
$$

Nota : Il existe diverses méthodes plus récentes qui ne donnent pas, pour autant, de résultats plus satisfaisants. 


\section{TECHNIQUES EXPÉRIMENTALES}

\subsection{Dispositif expérimental}

\subsubsection{Silo à fond plat}

Le silo à fond plat est représenté par une colonne qui est constituée de deux montants droits comportant une échelle graduée (de cinq centimètres en cing centimètres), qui sert de repère pendant le remplissage et la vidange.

Les dimensions du fut sont les suivantes:

- hauteur: $1,80 \mathrm{~m}$ :

- longueur: 0,30 ;

- profondeur: $0,15 \mathrm{~m}$.

Pour permettre une étude cinématique, le silo possède, en son fond plat, cing ouvertures permettant l'écoulement des rouleaux. La sélection du nombre d'ouvertures $(0$ à 5$)$ et l'agrandissement de l'ouverture (de 0 à $2 \mathrm{~cm}$ ) sont réalisés grâce à des plaques coulissantes contenant respectivement $2,3,4$ et 5 orifices.

\subsubsection{Silo muni d'une trémie}

En ce qui concerne le silo à trémie, il est représenté par deux montants verticaux de même dimension que ceux du modèle à fond plat. Les trémies sont matérialisées par des plaques calées dans le silo en quatre points : les inclinaisons possibles sont : $15^{\circ}, 30^{\circ}$. $45^{\circ}$ et $60^{\circ}$.

Afin d'avoir des repères fixes dans le massif, un cadre métallique a été réalisé sur lequel un maillage est matérialisé par des carrés de $5 \times 5 \mathrm{~cm}$.

\subsection{Mise en place du matériau}

Les rouleaux sont déposés dans le massif sous l'effet de la pesanteur, les couches successives ne sont pas compactées mais simplement disposées de façon à ce que les génératrices des rouleaux soient bien parallèles entre elles. Signalons que ce matériau est très peu compressible sous pression isotrope, la densité initiale de mise en place est constante et voisine de $d=1,11$. Cela permet d'avoir une bonne répétitivité dans l'assemblage, ce qui n'est pas le cas d'autres produits pulvérulents.

Or. on sait bien que pour un milieu granulaire donné. l'empilement des grains est très aléatoire et ses propriétés locales varient considérablement d'une région à une autre du massif, et également entre plusieurs reconstitutions d'un même empilement.

On ne peut considérer deux empilements successifs comme identiques que si leur porosité movenne ou leur densité en vrac sont égales. Ces paramètres sont implicitement définis en pratique comme des moyennes volumiques et, dans la plupart des cas, elles sont utilisées comme variables locales, mais l'inconvénient de cette définition est qu'en général, la moyenne spatiale d'une variable physique dépend du volume de référence choisi. Pour obtenir des essais comparables. il faut que les empilements de rouleaux conservent des conditions globales identiques. Cette manière de procéder est à la base de l'approche probabiliste.

On s'est attaché dans ces expériences à reproduire des massifs équivalents. On a eu tout de même de la dispersion due au caractère aléatoire que l'on a essayé de réduire au maximum en réalisant la même mesure plusieurs fois.

En effet, on sait que si on effectue la mesure un nombre de fois suffisamment grand suivant un processus à chaque fois identique, la moyenne statistique de la grandeur mesurée tendra vers sa moyenne probabiliste. Ceci a été vérifié dans le cas de l'étude statistique qui a été réalisée (1), qui consiste à étudier la répartition des contraintes au sein du massif.

\subsection{Dispositif de mesure des contraintes}

On a utilisé un appareil de mesure de contrainte basé sur le concept de GOURVES et FAUGERAS, et mis en place une chaîne d'acquisition automatique de mesures aboutissant directement à la création d'un fichier de valeurs sur micro-ordinateur. Ce dernier permet d'effectuer toute sorte de dépouillement.

Il s'agit d'un capteur de force référencé "TME FGTC », son étendue de mesure est de $0-5$ daN, il est raccordé à un coffret que gère éventuellement une imprimante.

Ce capteur délivre un signal électrique proportionnel à la pression subie, que le coffret met en forme en vue de son exploitation. L'ensemble assure les fonctions suivantes:

- possibilité de mesure et de mémorisation de force relative :

- gestion d'une liaison informatique pour raccordement à un micro-ordinateur :

- recopie analogique proportionnelle à la pression mesurée :

- indication du dépassement de l'échelle:

- affichage numérique de la pression avec indication de mesure et mémorisation de la valeur maximale atteinte selon l'échelle ou les échelles choisies.

\subsection{Mise en ouvre des essais}

\subsubsection{Etude statique}

Une seule et même procédure fut suivie pour réaliser tous les essais. Elle consiste à rechercher la répartiton des pressions isotropes au sein du massif en fonction de la profondeur: sur chaque carré de la surface qui a été discrétisée, on relève la force nécessaire pour déplacer un certain nombre de rouleaux selon leur axe longitudinal selon le mode opératoire suivant :

- on met en place les rouleaux jusqu'à une hauteur bien déterminée:

- on applique la charge verticale $Q$ :

- on pousse avec le capteur bien perpendiculairement au massif sur un ensemble de rouleaux jusqu'à déplacement de ceux-ci :

- on relève la force;

- on remet à zéro l'afficheur du conditionneur avant de passer au carré voisin ;

- une fois un passage complet effectué, on décharge le système et on foisonne le massif pour éliminer les concentrations de contraintes.

On répète ces opérations plusieurs fois afin d'avoir un nombre de mesures suffisant pour permettre une analyse statistique satisfaisante. 


\subsubsection{Etude cinématique}

Pour chaque manipulation, les modèles ont été remplis de la même manière et sur une hauteur identique. L'observation d'ensemble du matériau analogique est effectuée à l'aide de couches de rouleaux colorés, espacées de $5 \mathrm{~cm}$. Pendant l'écoulement, des photos sont prises toutes les secondes.

Principe de la méthode : on suit, lors de l'écoulement filmé en vidéo, l'évolution de quelques rouleaux au sein du modèle bidimensionnel à paroi lisse ou rugueuse, à fond plat ou à trémie avec un orifice centré ou excentré, on analyse image par image et on en déduit :

- les déplacements des grains et leurs vitesses instantanées selon leurs positions (X, Y) dans le silo: - l'évolution globale du massif.

En utilisant un programme adéquat, on obtient automatiquement les déplacements relatifs de ces points par rapport à la moyenne des déplacements du même cliché, et on obtient également le graphe des vitesses relatives de différents points par rapport à la vitesse moyenne sur l'intervalle de temps considéré.

\section{COMPARAISON ENTRE LA MÉTHODE DE JANSSEN ET LA MÉTHODE EXPÉRIMENTALE}

Les comparaisons des différentes courbes obtenues sont indiquées sur les figures 2 et 3 .

On constate que la méthode expérimentale donne en général des efforts plus élevés que ceux obtenus théoriquement. Cependant, cette différence a tendance à disparaître au fur et à mesure que la profondeur augmente dans le cas où la surface libre est non chargée (fig. 2), la courbe expérimentale ne présente pas de différence notable avec celle obtenue par la méthode de JANSSEN.

En revanche, sur la figure 3 qui représente la comparaison entre les courbes théorique et expérimentale dans le cas où la surface libre est chargée, on constate qu'il existe, à la partie supérieure de la colonne, une très grande différence entre les valeurs expérimentales et les valeurs théoriques, ces dernières étant très faibles.

Mais, à partir d'une hauteur $\mathrm{H}=50 \mathrm{~cm}$ cette différence diminue nettement et disparait à une hauteur $\mathrm{H}=120 \mathrm{~cm}$. Ces résultats sont analogues à ceux obtenus par MEZGHANI à I'Université de ClermontFerrand. Cette différence est due à la répartition non régulière de la charge dans la partie haute du silo.

\section{EFFET DE PAROIS}

5.1. Etude des trajectoires des particules : $\mathrm{Z}=\mathrm{f}(\mathrm{t})$

On a rempli, pour chaque expérience, le modèle à la même hauteur $(Z=50 \mathrm{~cm})$, on repère alors un certain nombre de particules colorées en notant leurs coordonnées de départ. Ensuite, après déclenchement du chronomètre au moment où l'orifice est ouvert, on suit en temps réel le mouvement des particules.

Des écoulements dans des modèles munis de parois semi-rugueuses et de parois rugueuses ont été réalisés. Le mouvement du rouleau repéré est suivi sur un écran plat à partir de sa position initiale $\left(\mathrm{X}_{0}, \mathrm{Z}_{0}\right)$ jusqu'à la sortie de l'orifice de vidange.

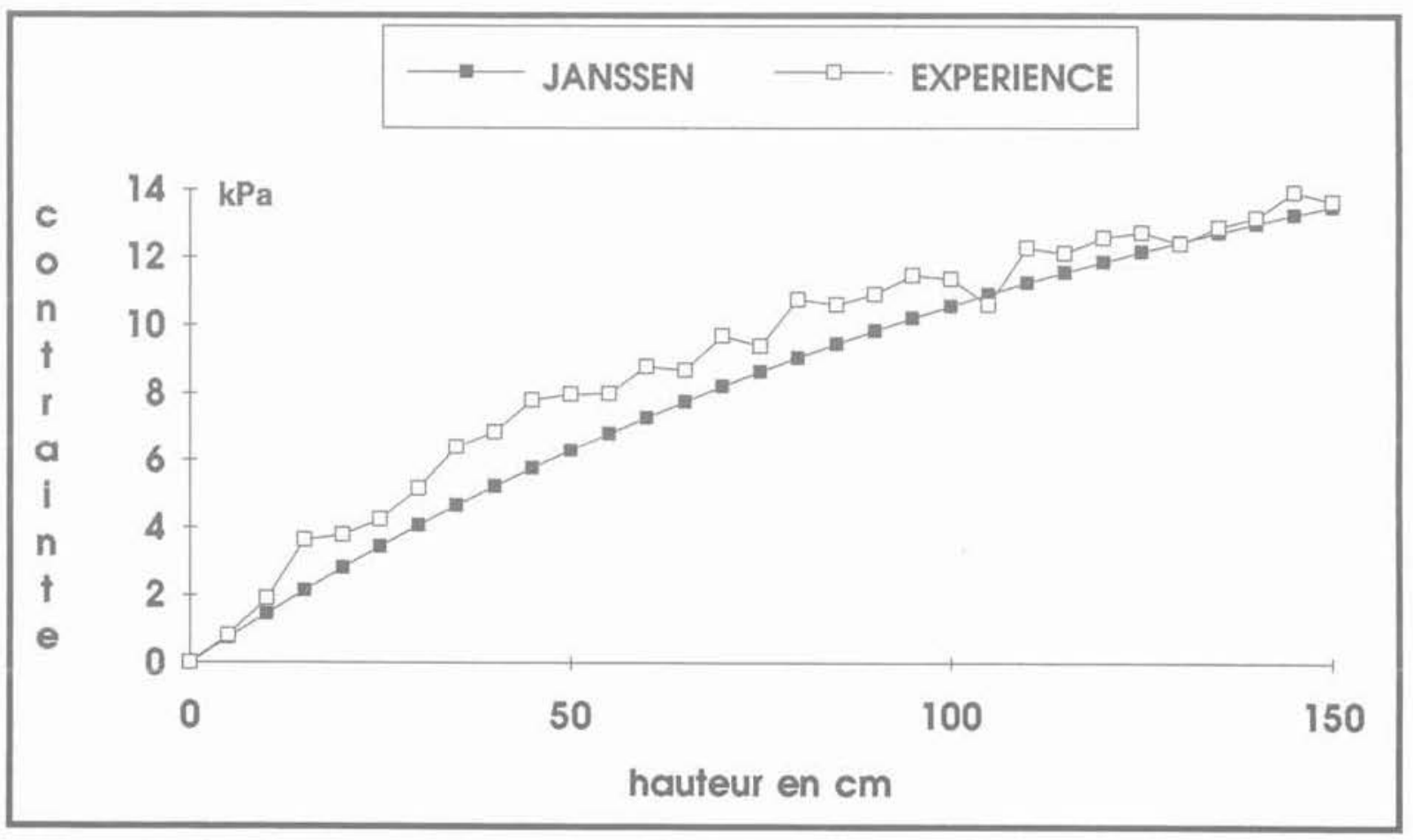

Fig. 2, - Comparaison des méthodes théorique et expérimentale (surface libre non chargée).

Fig. 2. - Comparison betweenn the experimental and theoretical methods (boundary free of charge). 


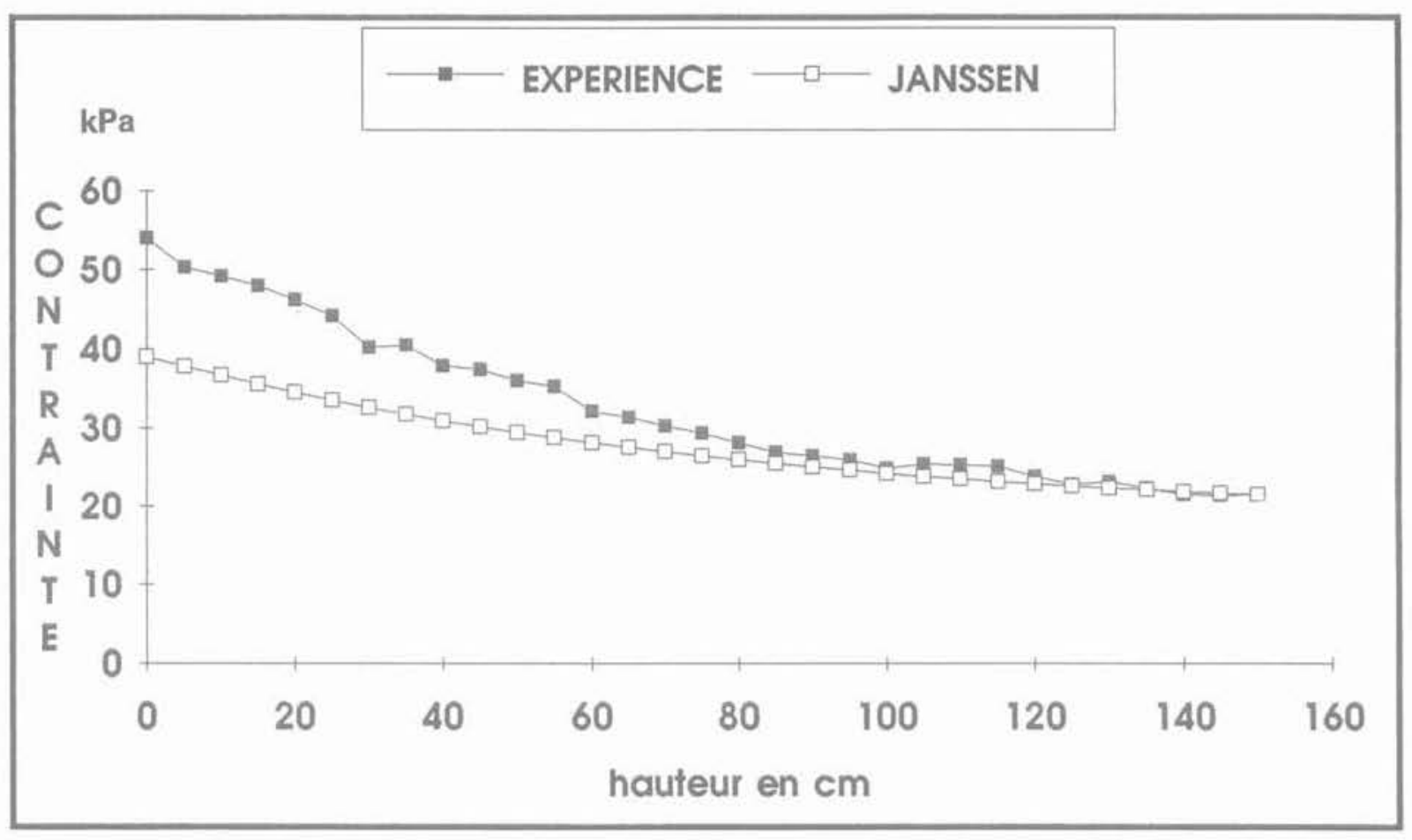

Fig. 3. - Comparaison entre les courbes théorique et expérimentale (surface libre chargée).

Fig. 3. - Comparison between experimental and theoretical curves lboundary submitted to chargest.

Ainsi, pendant les 18 premières secondes d'écoulement, on a relevé toutes les deux secondes la valeur de la côte $Z$. Après 18 secondes les valeurs de la côte $\mathrm{Z}$ ont été notées toutes les secondes. Dans tous les essais réalisés, les rouleaux se sont écoulés selon le mode dit " de cheminée ", la dimension de l'orifice étant constante.

\subsubsection{Silo à fond plat et à parois semi-rugueuses}

Les courbes des figures 4 à 7 représentent l'évolution des particules au cours du temps: ainsi la figure 4 est relative aux particules repérées au voisinage des parois. La figure 5 correspond aux particules repérées dans la zone intermédiaire. La figure 6 représente l'évolution des particules dans l'axe de la colonne. Les courbes des figures 4,5 et 6 montrent que les particules dans les trois zones évoluent dans un fuseau assez restreint. Ceci s'explique par le fait que les grains sont animés d'une même vitesse dans chaque zone.

Si l'on compare la trajectoire d'un grain au voisnage des parois avec les deux autres trajectoires relatives, respectivement à un grain de l'axe de la colonne et à un grain de la zone intermédiaire (fig. 7), on constate que les grains évoluent dans un même faisceau jusqu'à une certaine hauteur $\mathrm{Z}=35 \mathrm{~cm}$, puis les trois courbes divergent. Ceci s'explique par le fait que les grains doivent changer de direction au voisinage des parois lorsqu'ils atteignent la zone de transition pour s'orienter vers l'orifice de vidange, ce qui provoque un certain ralentissement par rapport aux deux autres. Ce phénomène est évidemment très accentué dans le cas d'un orifice excentré pour les grains qui se trouvent de l'autre côté de l'orifice.

\subsubsection{Silo à fond plat et parois rugueuses}

Le même principe que précédemment a été utilisé. Ainsi les courbes des figures 9 et 10 montrent que dans ces deux zones la vitesse reste sensiblement constante, puisque les grains évoluent dans un faisceau très resserré. Une première conclusion est que dans ces deux zones les mouvements des grains au sein du massif ne sont pas perturbés par la présence de parois rugueuses. Par ailleurs, au voisinage des parois les trajectoires des grains ne sont plus dans un même faisceau, mais ces dernières sont indépendantes les unes des autres.

Ceci montre que dans cette zone latérale, le mouvement des grains est très perturbé par la rugosité des parois. Ce phénomène s'accentue au fur et à mesure qu'on se rapproche de celle-ci, cette augmentation de la perturbation se fait sentir différemment sur la partie haute du modèle.

Le figure 11 montre clairement ce phénomène : elle présente une comparaison entre l'allure des différentes trajectoires obtenues dans les trois zones du modèle. Toutefois, on note que l'évolution des déplacements des grains en fonction du temps, à la base supérieure de la paroi du modèle, après une vidange centrée, semble indiquer qu'en début de vidange il se produit un certain ralentissement. Ceci est également discernable, comme on le verra par la suite, sur les mesures de déplacements faites au voisinage des parois.

En conclusion, nous pouvons dire que les différences relevées entre les trajectoires des particules des différentes zones peuvent être imputées, non seulement à la position et à la dimension de l'orifice de vidange mais surtout à la nature de la paroi. En fait, 


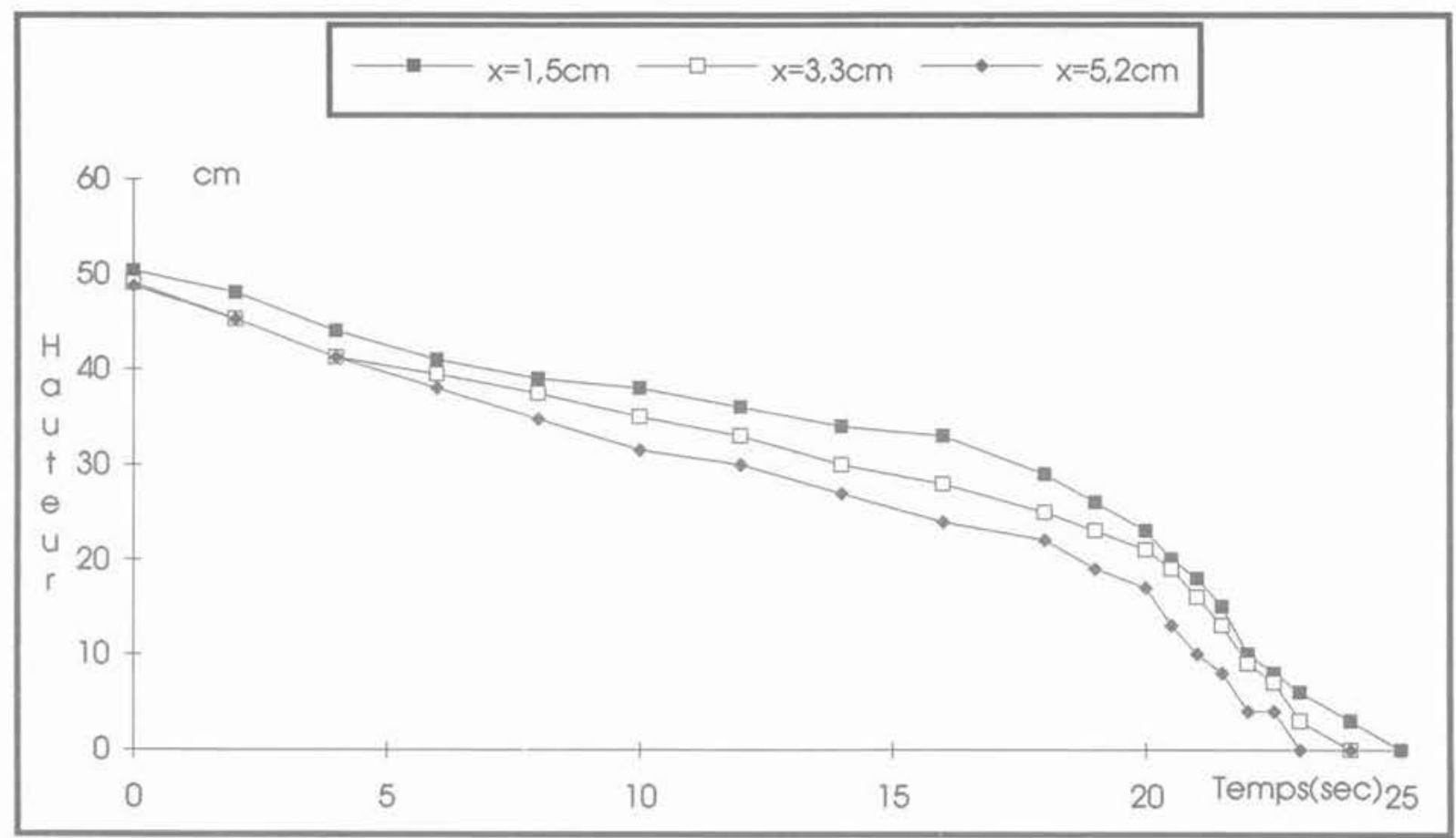

Fig. 4. - Trajectoires des particules au voisinages des parois semi-rugueuses.

Fig. 4. - Trajectories of particles in the vicinity of the semi-wrikled walls.

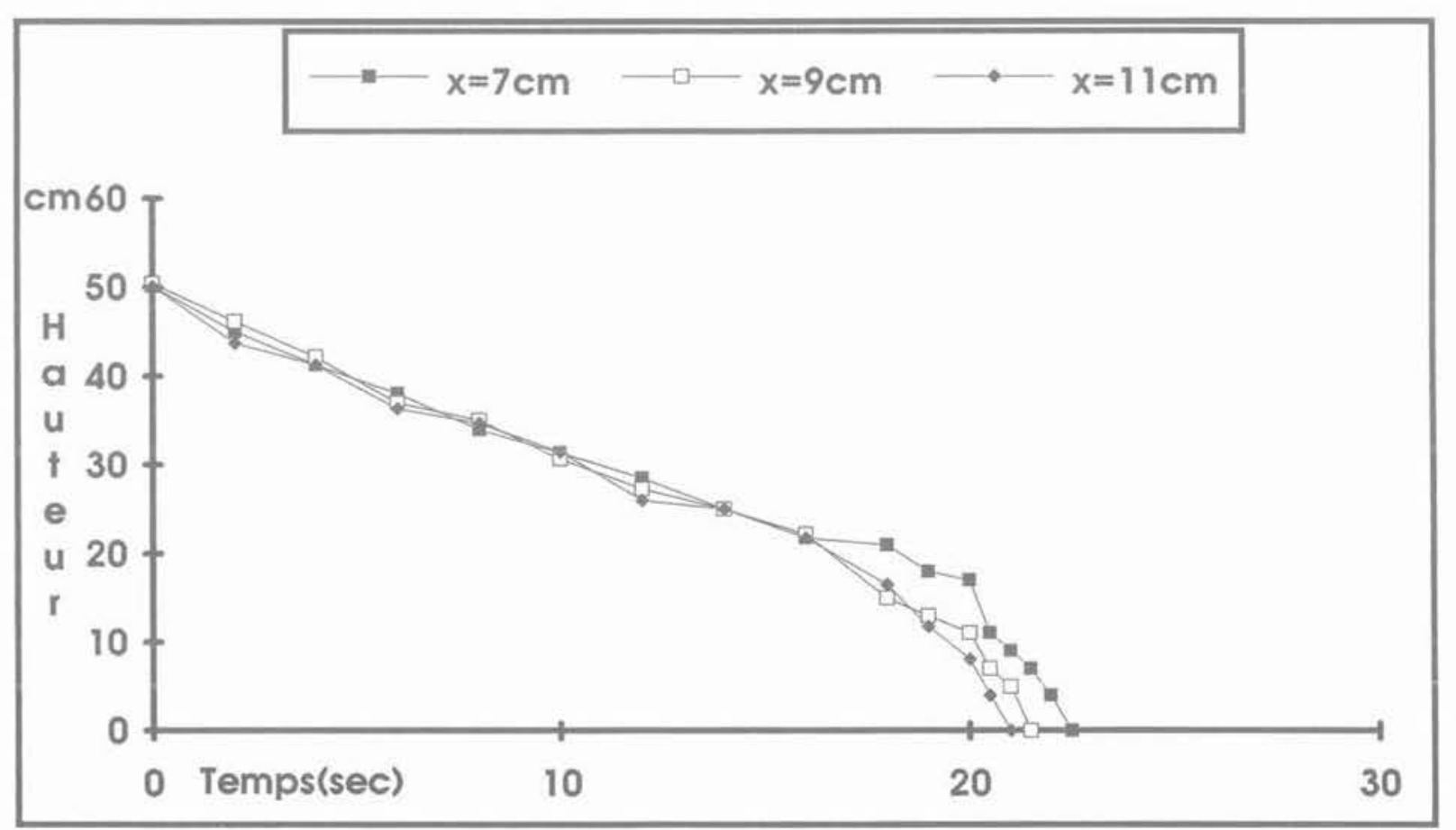

Fig. 5. - Trajectoires des particules de la zone intermédiaire (parois semi-rugueuses).

Fig. 5. - Trajectories of particles of the intermediate region (semi-wrinkled walls). 


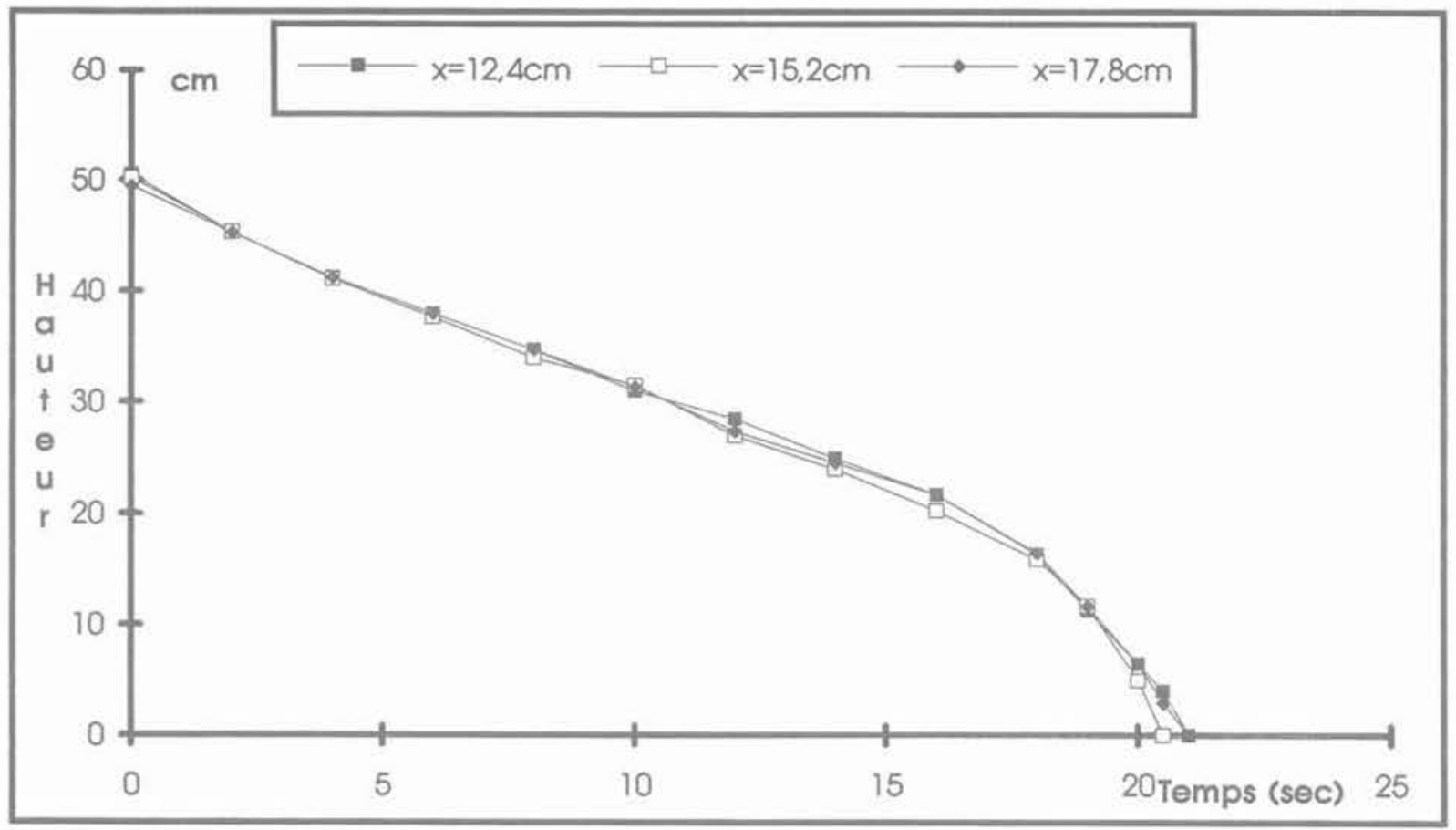

Fig. 6. - Trajectoires des particules de l'axe de la colonne (parois semi-rugueuses).

Fig. 6. - Trajectories of particles situated on the axes of the column (semi-wrinkled walls).

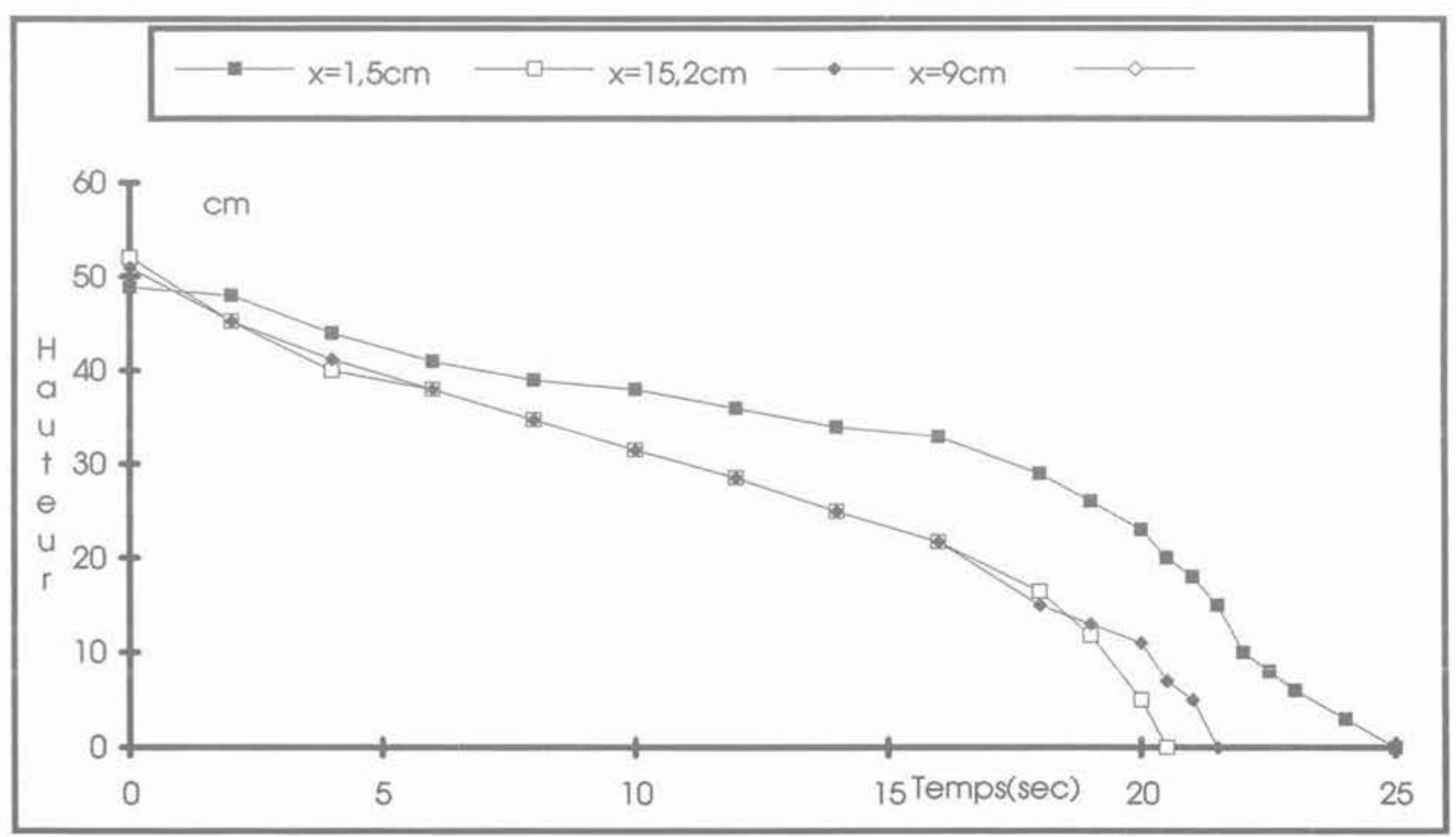

Fig. 7. - Comparaison entre les trajectoires des particules des trois zones (parois semi-rugueuses). Fig. 7. - Comparison of the trajectories of particles situated in each one of the regions (semi-wrikled walls). 


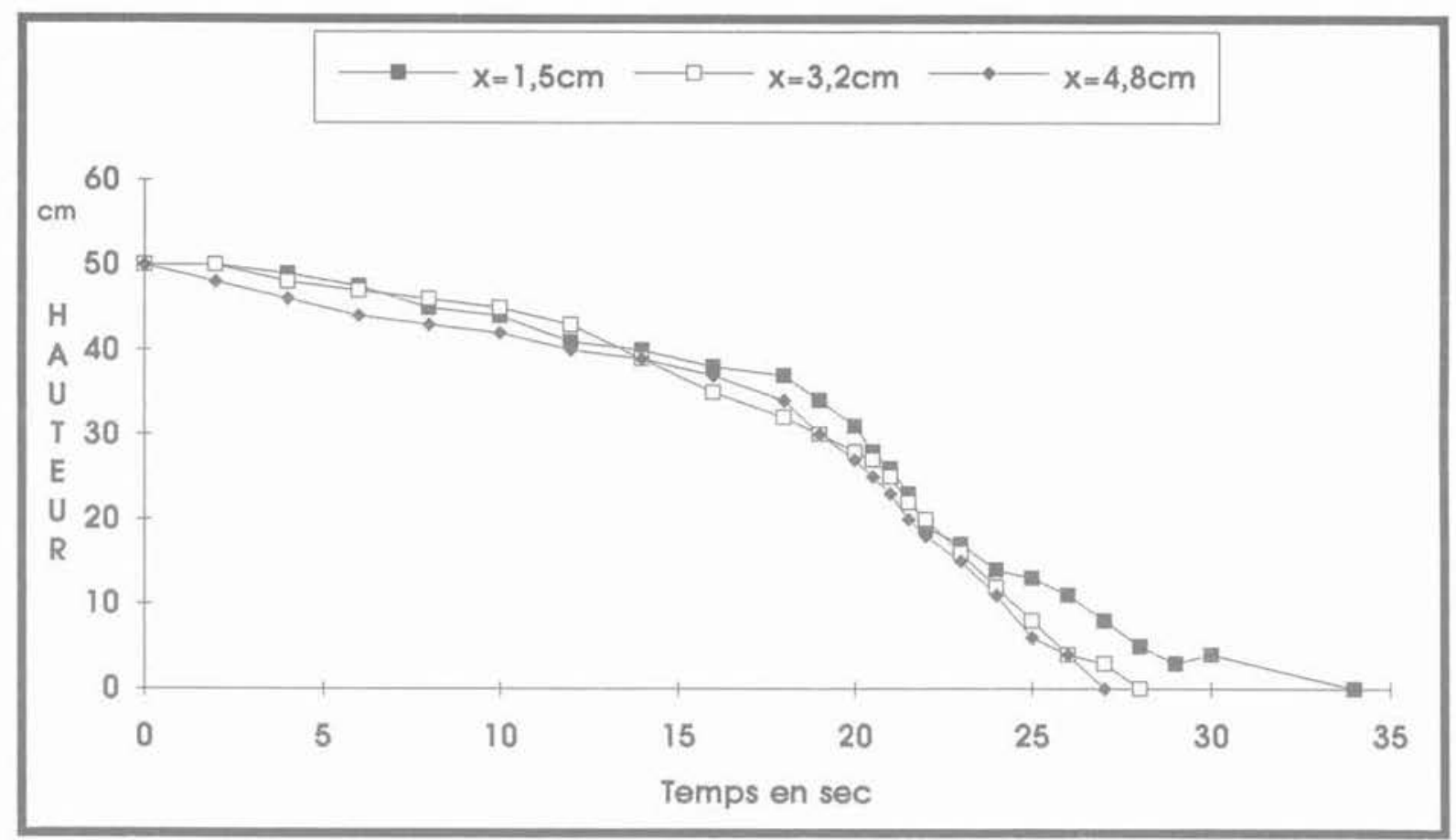

Fig. 8. - Trajectoires des particules au voisinage des parois (parois rugueuses).

Fig. 8. - Trajectories of particles located in the vicinity of wrinkled walls.

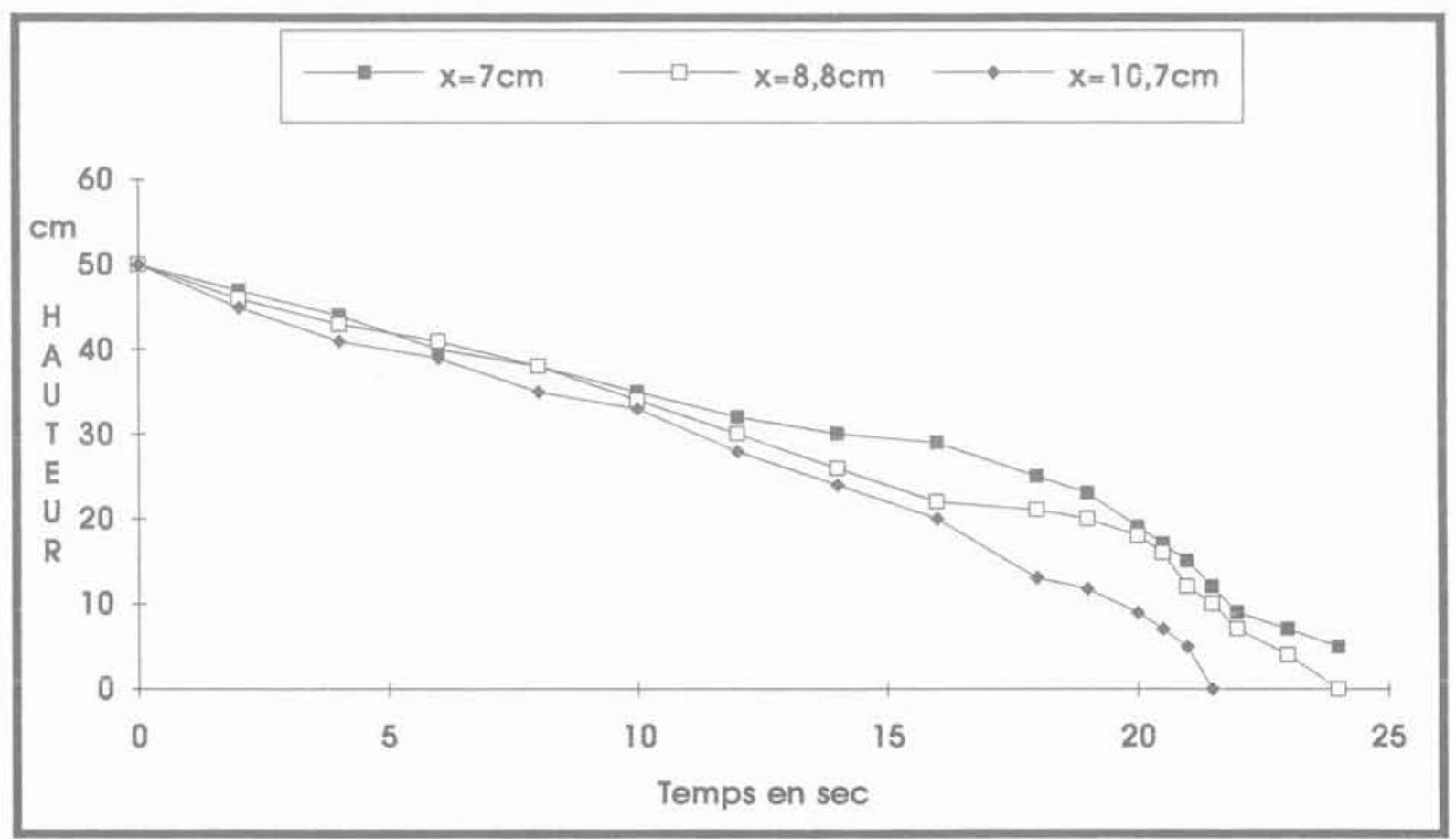

Fig. 9. - Trajectoires des particules de la zone intermédiaire (parois rugueuses).

Fig. 9. - Trajectories of particles of the intermediate region (in the case of wrinkled walls) 


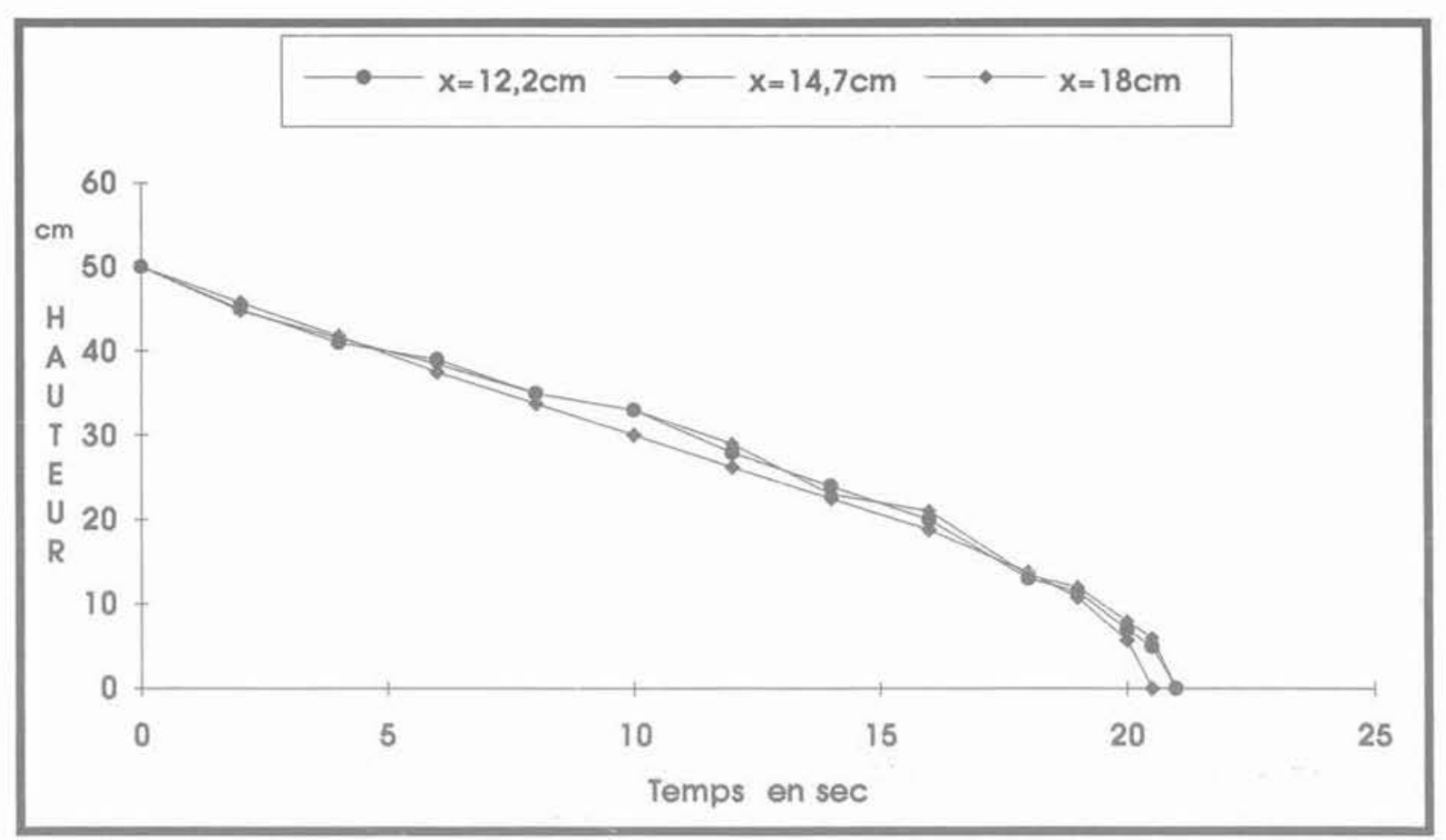

Fig. 10. - Trajectoire des particules de l'axe de la colonne (parois rugueuses).

Fig. 10. - Trajectories of particles located on the axes of the column (wrinkled walls).

les grains au contact ou à proximité de la paroi rugueuse mettent plus de temps pour atteindre l'orifice de vidange, environ 35 secondes (fig. 11), alors que ce temps est de l'ordre de 24 secondes pour les grains qui se trouvent à proximité des parois semirugueuses (fig. 7) ; donc la nature des parois provoque des modifications relativement importantes du comportement local du matériau ensilé.

\subsection{Influence de la rugosité sur le mode d'écoulement}

Les résultats expérimentaux concernant l'écoulement à travers un orifice dans un modèle à parois rugueuses ou semi-rugueuses et à trémie inclinée de $45^{\circ}$ et $60^{\circ}$ sont présentés ici. Les mesures et les résultats obtenus au cours de ces essais représentent une

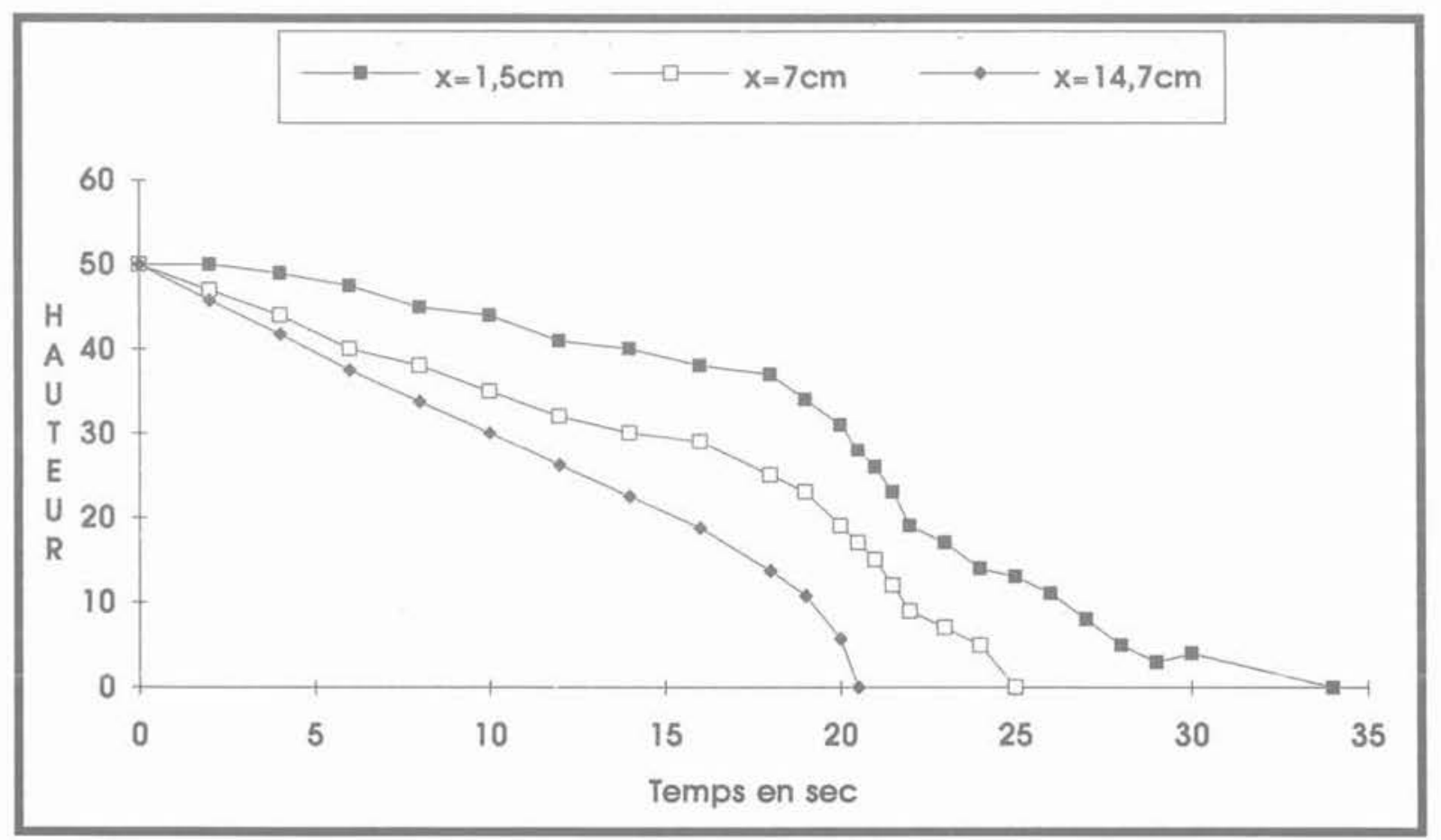

Fig. 11. - Comparaison entre les trajectoires des particules des trois zones (parois rugueuses).

Fig. 11. - Comparison between the trajectories of particles of the three regions lin the case of wrinkled walls). 
masse de données considérable dont l'exploitation et le traitement ont conduit aux interprétations et aux conclusions présentées ci-dessous.

\subsubsection{Etude qualitative}

Au cours de ces expériences on a distingué, en géné$\mathrm{ral}$, deux types d'écoulement : écoulement en masse et écoulement en cheminée. De plus, dès le début de la vidange, tout le matériau descend régulièrement et on observe un mouvement d'ensemble du matériau ; c'est ce que P'on observe généralement dans les silos prototypes ou en grandeur réelle, munis de trémies lisses et suffisamment inclinées. En ce qui concerne le débit massique, on constate expérimentalement, que pour un orifice de diamètre donné et une paroi latérale de rugosité bien déterminée, celui-ci augmente rapidement avec l'inclinaison et diminue légèrement avec la rugosité. Nous distinguons dans le massif en mouvement deux parties nettement différentes :

- dans la partie basse où lon distingue, dans le cas de la trémie $45^{\circ}$, deux zones. Une première zone où les rouleaux situés près des parois inclinées s'orientent vers le milieu de la trémie, une deuxième où les rouleaux sont en écoulement :

- dans la partie haute, on constate que les particules se mettent en mouvement avec une vitesse moindre près des parois, en raison du frottement de celles-ci.

Dans le cas où la trémie est inclinée de $60^{\circ}$, les mêmes constatations que précédemment peuvent être faites à une particularité près: la zone d'orientation des rouleaux disparait, aussi bien pour des parois rugueuses que pour des parois semi-rugueuses. La disparition de cette zone est imputable essentiellement à l'inclinaison de la trémie qui est d'ailleurs très supérieure à l'angle de frottement grains/grains.

Ces résultats montrent que le brusque passage de l'état d'équilibre de la matière ensilée au repos à celui de la matière en mouvement par suite de la vidange entraîne un déséquilibre total de la masse ensilée; la plupart des auteurs admettent que cela provoque généralement des augmentations importantes et rapides de la poussée des grains, assimilant ces dernières à des effets dynamiques qui sont actuellement encore mal définis. Les expériences ainsi réalisées ont mis en évidence des phénomènes pressentis jusqu'ici mais insuffisamment définis de façon descriptive.

Comportement de la surface libre du massif :

Dans le cas des parois semi-rugueuses, labaissement de la surface libre en fonction du temps est très régulier, sauf au début de l'écoulement où la surface libre du massif n'amorce sa descente que quelques secondes après l'ouverture de l'orifice. Cet effet différé résulte de l'existence d'un frottement interne qui entraîne une lente propagation du cisaillement dans la masse ensilée. En fait, la surface libre reste plane jusqu'à une certaine hauteur $\mathrm{Z}$ bien déterminée, fonction de l'inclinaison de la trémie. Alors que, dans le cas des parois rugueuses, c'est le phénomène contraire qui se produit.

En effet, quelques secondes après l'ouverture de l'orifice, on remarque un affaissement de la surface libre

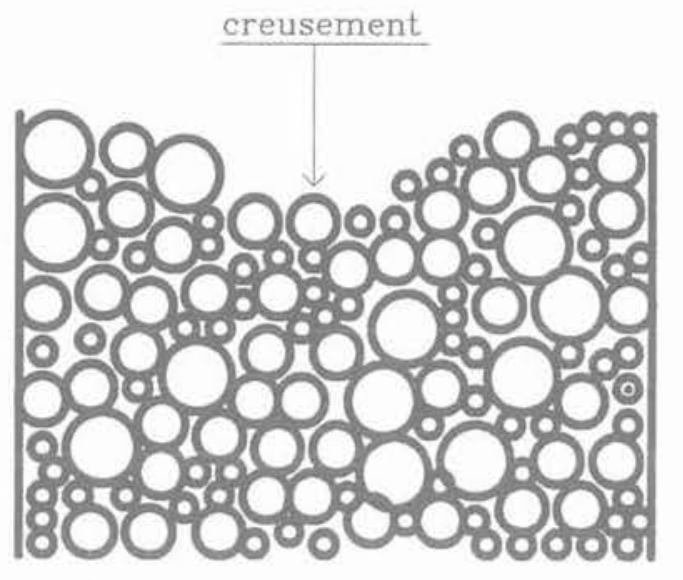

$t=2 s$

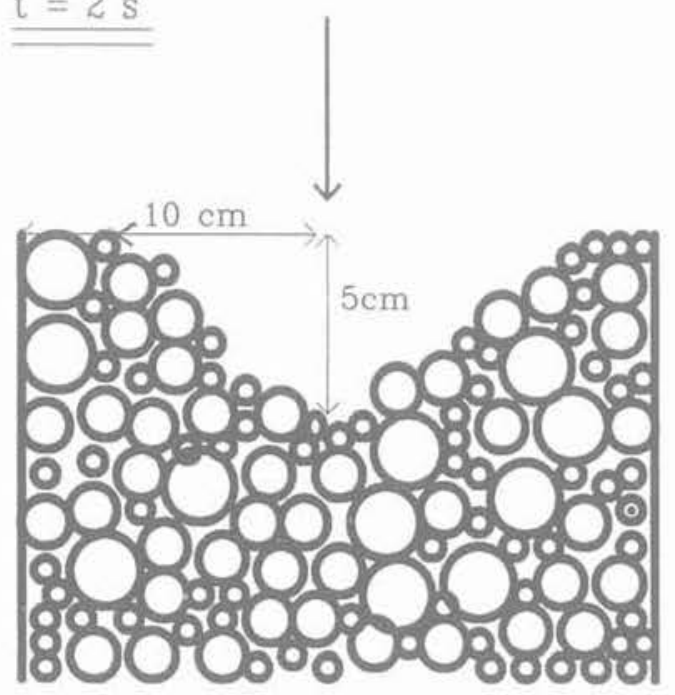

$\mathrm{t}=15 \mathrm{~s}$

Fig. 12. - Comportement de la surface libre (parois rugueuses).

Fig. 12. - Evolution of the free boundary (in the case of wrinkled walls).

et, au fur et à mesure que l'écoulement s'établit au sein du silo, il se développe progressivement un cône faisant avec l'horizontale un certain angle (fig. 12) qui évolue avec le temps.

L'observation attentive du film tourné au cours de l'écoulement a permis de constater, en fait, que les grains à proximité des parois rugueuses sont freinés et s'immobilisent pendant un certain temps en restant comme collés à la paroi. Mais, une fois que la surface libre initiale dépasse ce niveau, les grains sont relâchés par la paroi et glissent sur cette dernière en formant un angle d'inclinaison égal à celui du frottement grain/grain. Ceci montre bien que la rugosité de la paroi fait passer la vidange au sein du silo de la vidange en semi-masse à la vidange en cheminée. Ces effets de parois peuvent causer des changements non négligeables au niveau du comportement général du massif au cours de l'écoulement dans la mesure où ils peuvent modifier le fonctionnement de la vidange et, de ce fait, changer l'état de contrainte sur les parois. 


\subsubsection{Etude quantitative}

Grâce à l'enregistrement vidéo et aux diapositives, on a pu déterminer les déplacements des particules entre deux images prises dans un intervalle de temps égal à deux secondes.

Dans le cas de la trémie $45^{\circ}$ avec parois semirugueuses, si on considère, dans la partie haute du silo, des particules sur un même niveau à un temps $t$, on les retrouve au même niveau au temps $t+$ $2 \mathrm{~s}$; cela veut dire que les particules qui se trouvent dans la partie haute du modèle sont animées d'une même vitesse, sauf les grains qui se trouvent à proximité de la paroi. Ceci montre qu'il y a peu de déplacements relatifs entre les grains. Ainsi, les grains s'écoulent en engendrant un écoulement en bloc (fig. 13)

Dans le cas des parois rugueuses, les points situés sur une bande de $5 \mathrm{~cm}$ de long des parois latérales subissent un déplacement de deux à trois fois plus petit que les autres points qui se situent dans la zone centrale (fig. 15) ; dans la partie basse du silo, on observe une zone d'accélération au milieu de la trémie où les déplacements sont plus grands que la moyenne.

L'analyse numérique de ces résultats donne les graphes des vitesses relatives des différents points pour l'intervalle de temps considéré. Ainsi, par exemple,

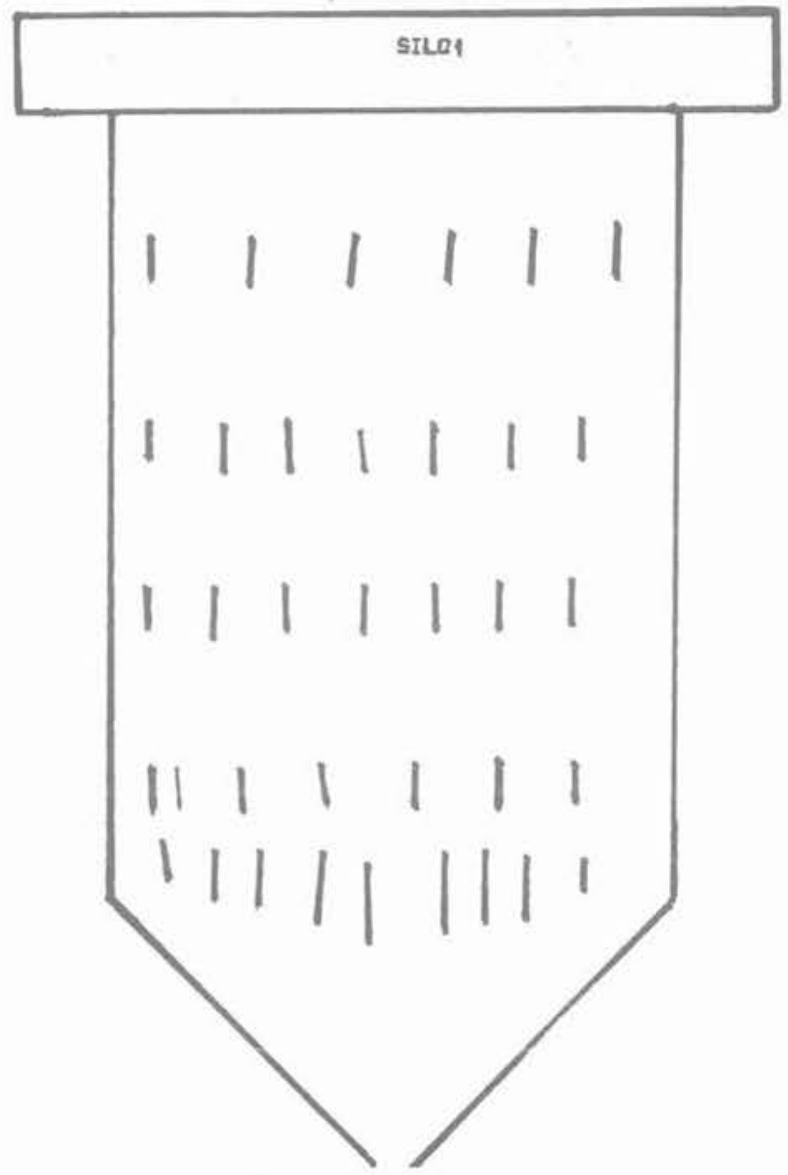

Fig. 13. - Déplacements entre photos 2 et 3 (parois semi-rugueuses).

Fig. 13 - Displacements from photo 2 to photo 3 (case of semi-wrinkled walls).

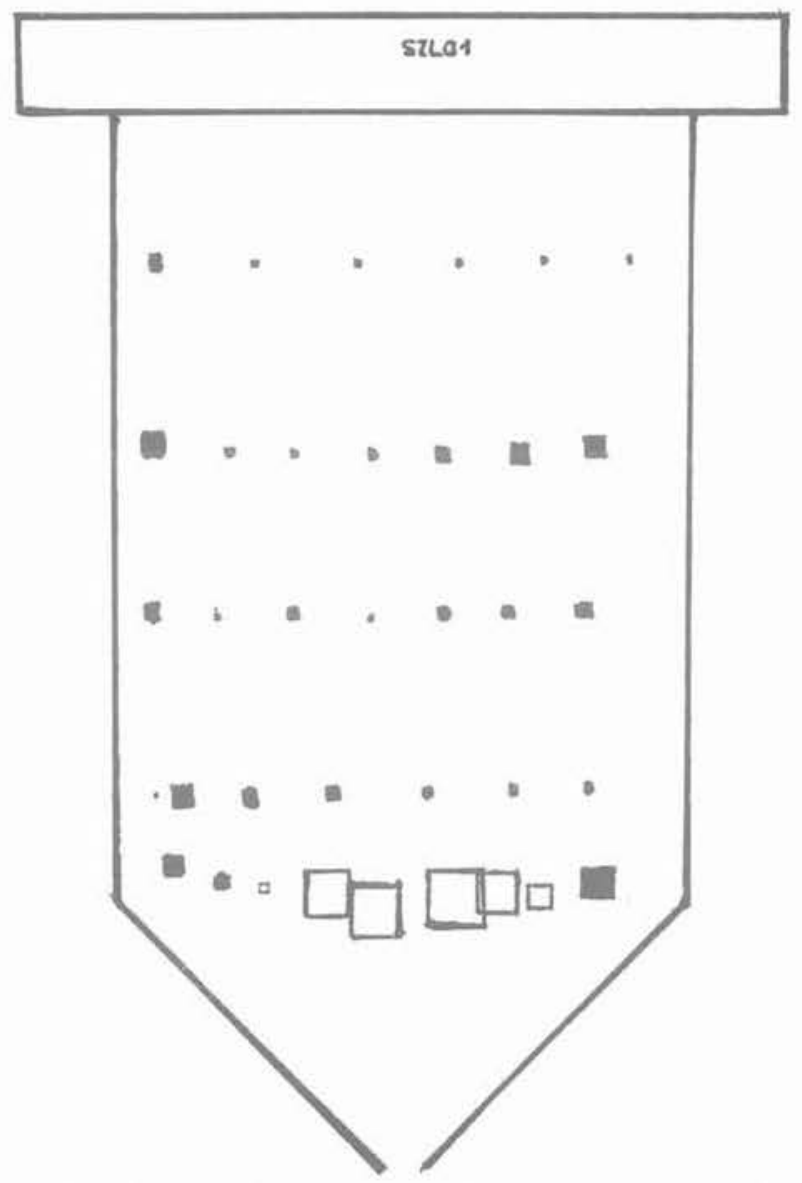

Fig. 14. - Déplacements relatifs entre photos 2 et 3 (parois semi-rugueuses).

Fig. 14. - Relative displacements from photo 2 to photo 3 (semi-wrinkled walls).

la figure 16 montre que sur les parois latérales rugueuses, les vitesses sont beaucoup plus faibles que la moyenne, ce sont les grands carrés ombrés. En revanche, quand on se déplace vers le milieu du silo, on observe sur cette figure (fig. 16) qu'on passe des carrés ombrés à des carrés clairs (blanc). Ceci confirme que la rugosité affaiblit la vitesse des grains au voisinage immédiat des parois verticales du modèle. La même analyse reste valable pour le modèle à trémie d'inclinaison $60^{\circ}$ à une différence près : en effet. on constate qu'au voisinage des parois de la trémie d'inclinaison $45^{\circ}$ les rouleaux s'orientent vers la zone de la chute libre par des déplacements parallèles à ces parois alors que dans le cas du modèle à trámie d'inclinaison $60^{\circ}$ nous n'observons plus ce phénomène.

Pour mieux mettre en évidence l'influence de la rugosité dans les deux modèles, on a relevé sur toutes les figures qui correspondent aux déplacements entre les clichés, les valeurs du déplacement maximal (Maxi), du déplacement minimal (Mini) et du déplacement moyen (Moyen) ; ainsi ;

- le tableau 1 présente les résultats relatifs au modèle à " trémie $60^{\circ}$ ";

- le tableau 2 correspond aux valeurs obtenues dans le modèle à " trémie $45^{\circ}$ n. 


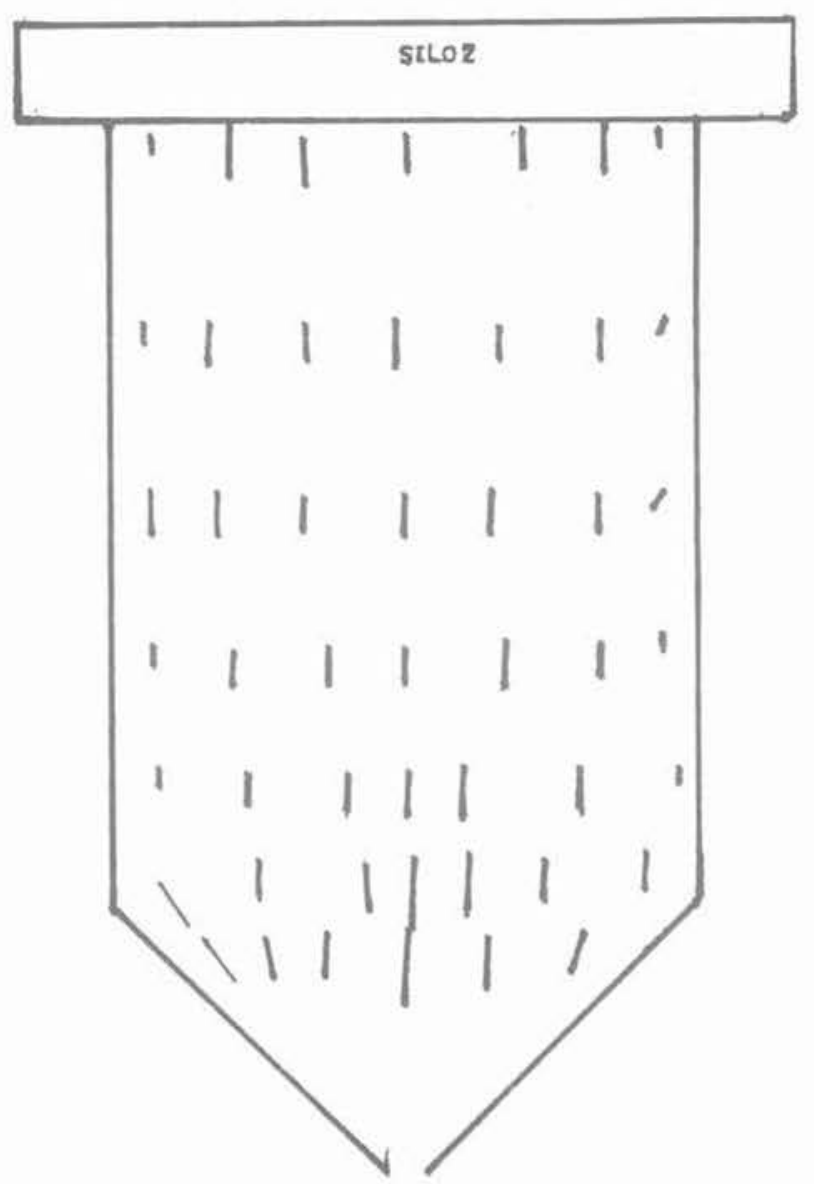

Fig. 15. - Déplacements entre photo 17 et 18 (parois rugueuses),

Fig. 15. - Displacements from photo 17 to photo 18 (wrinkled walls).

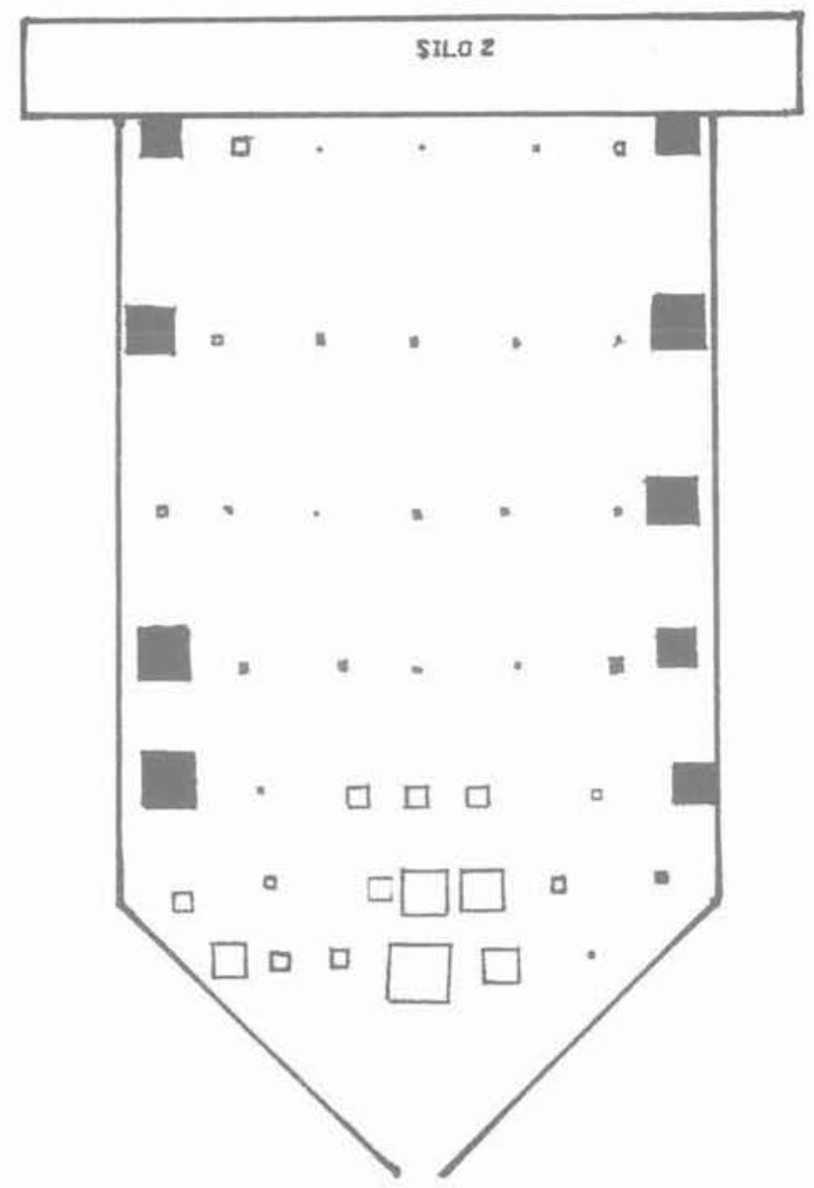

Fig. 16. - Déplacements relatifs entre photos 17 et 18 (parois rugueuses).

Photo 16. - Relative displacements from photo 17 to photo 18 (wrinkled walls).

Tableau 1. - Déplacements entre photos en $\mathrm{cm}$ (trémie $60 \%$ )

Table 1. - Displacements in centimeters (measured between photographs) in the case of a $60^{\circ}$ inclined hopper.

\begin{tabular}{|c|c|c|c|c|c|c|c|}
\hline \multicolumn{9}{|c|}{ Trémie $60^{\circ}$} \\
\hline \multicolumn{4}{|c|}{ Parois semi-rugueuses } & \multicolumn{5}{c|}{ Parois rugueuses } \\
\hline$N^{\circ}$ des photos & Maxi & Mini & Moyen & $N^{\circ}$ des photos & Maxi & Mini & Moyen \\
\hline 22 et 23 & 7,2 & 0,8 & 3,3 & 10 et 11 & 6,5 & 0,2 & 1,7 \\
\hline 23 et 24 & 7,8 & 1,8 & 3,3 & 11 et 12 & 7,1 & 0,3 & 2,6 \\
\hline 25 et 26 & 7,9 & 1,4 & 3,2 & 12 et 13 & 5,2 & 0,4 & 2,2 \\
\hline
\end{tabular}

Tableau 2. - Déplacements entre photos en $\mathrm{cm}$ (trémie $45^{\circ}$ ).

Table 2. - Displacements in centimeters (measured between photographs) in the case of a $45^{\circ}$ inclined hopper.

\begin{tabular}{|c|c|c|c|c|c|c|c|}
\hline \multicolumn{9}{|c|}{ Trémie $45^{\circ}$} \\
\hline \multicolumn{4}{|c|}{ Parois semi-rugueuses } & \multicolumn{5}{c|}{ Parois rugueuses } \\
\hline$N^{\circ}$ des photos & Maxi & Mini & Moyen & $N^{\circ}$ des photos & Maxi & Mini & Moyen \\
\hline 1 et 2 & 3,9 & 1,2 & 2 & 17 et 18 & 3,4 & 0,3 & 1,7 \\
\hline 2 et 3 & 4 & 1,5 & 2,4 & 18 et 19 & 4,8 & 0,3 & 1,8 \\
\hline 3 et 4 & 5,3 & 0,7 & 2,3 & 19 et 20 & 8 & 0,3 & 2,2 \\
\hline
\end{tabular}


Ces tableaux montrent d'une part que les déplacements minimaux sont plus grands dans le modèle à parois rugueuses. Certaines particules sont pratiquernent arrêtées au voisinage des parois (déplacements « Mini * ayant comme valeurs 0,6 et 0,8 entre deux photos) D'autre part, les déplacements moyens sont toujours supérieurs dans les modèles à parois semi-rugueuses.

Ces derniers montrent également qu'au fur et à mesure que l'inclinaison de la trémie augmente, les déplacements maximaux augmentent ainsi que la vitesse moyenne, que ce soit au sein d'un modèle à parois rugueuses ou à parois semi-rugueuses.

Au terme de cette analyse, et compte tenu de tous les résultats obtenus jusqu'ici, on peut proposer deux modes de mécanisme d'écoulement au sein des modèles étudiés, ceci en décrivant le champs de vitesse au sein du silo dans plusieurs zones dont le nombre dépend de la rugosité des parois et de l'inclinaison de la trémie (fig. 17 et fig, 18).

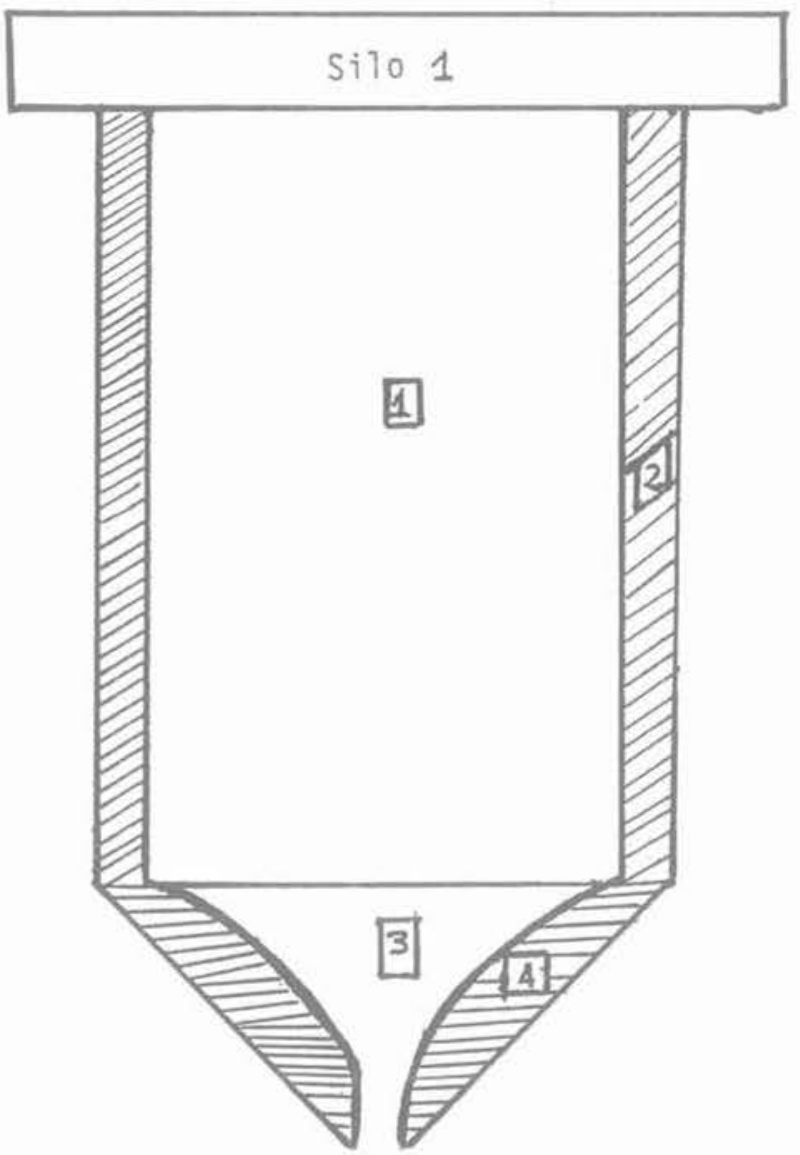

Fig. 17. - Différentes zones d'écoulement dans le modèle á trémie $45^{\circ}$ (parois rugueuses).

Zone 1: zone de vitesse quasiment constante.

Zone 2: zone de vitesse minimale, I'influence des parois sur une bande d'environ $4,5 \mathrm{~cm}$. Zone 3 : zone d'accélération

Zone 4 : zone d'orientationn des rouleaux vers la zone de chute libre.

Fig. 17. - Some flow regions for a model with an hopper inclined of $45^{\circ}$

First region : velocity quasi constant. Second region : minimal velocities due to the wall influence in a band of $4.5 \mathrm{~cm}$ thickness.

Third region: acceleration region. Fourth region : orientation of rolls towards the region of free motion.

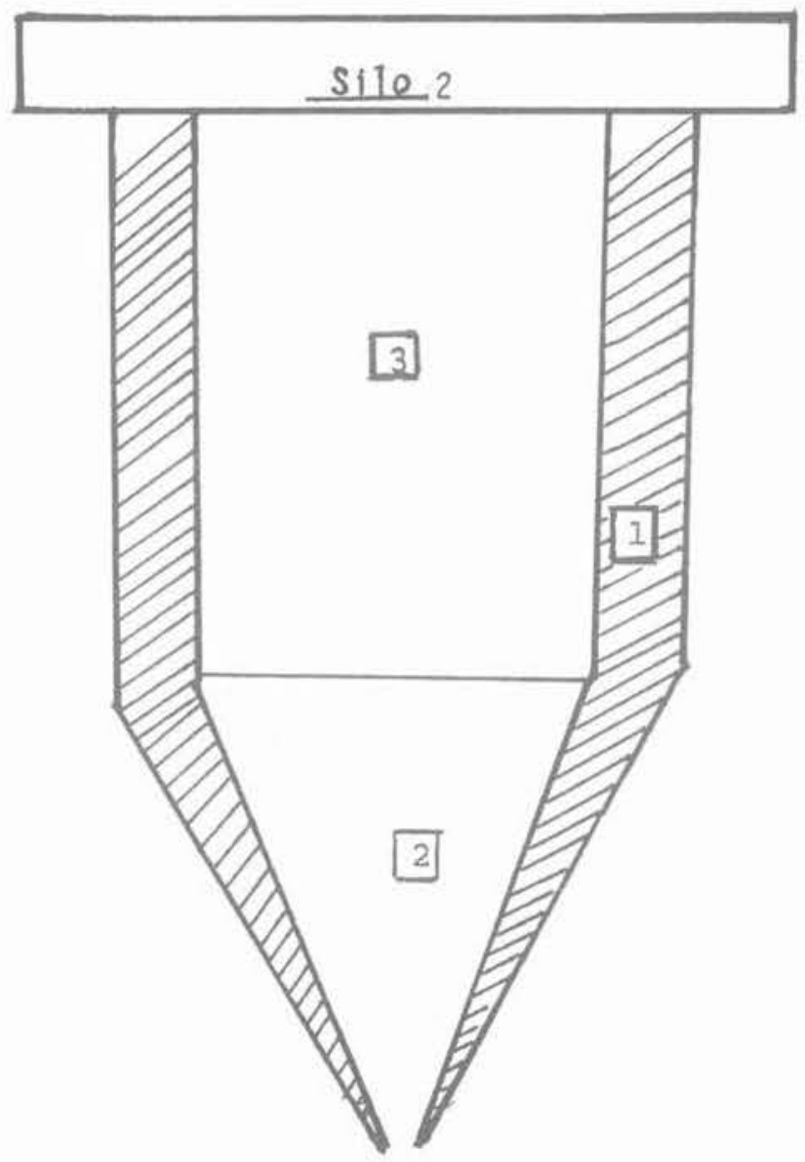

Fig. 18. - Différentes zones d'écoulement dans le modẻle à trémie $60^{\circ}$ (parois rugueuses).

Zone 1: zone de vitesse minimale, influence maximale de la paroi. Zone 2 : zone d'accélération, les points atteignent une vitesse maximale. Zone 3 : zone où le massif se déplace avec une même vitesse en tout point.

Fig. 18. - Some flow regions for a model with à $60^{\circ}$ inclined hopper (wrinkled walls).

First region : region of minimal velocity and maxima: influence of the wall. Second region : region where acceleration takes place, the particles reaching a maximal velocity

Third region: in which the granular material has a rigid motion (the same velocity for the particles)

\subsection{Etude statique}

Pour mettre en évidence linfluence de la rugosité au niveau statique, on a tout d'abord effectué un certain nombre de mesures de la contrainte moyenne au sein de deux modèles, l'un à parois rugueuses, lautre à parois lisses, dans les trois zones suivantes :

- au voisinage des parois ;

- dans la zone intermédiaire :

- sur l'axe de la colonne.

Ensuite, on a tracé diverses courbes se rapportant aux résultats qui ont semblé les plus importants. Ainsi, la figure 19 présente une comparaison, dans le cas des parois semi-rugueuses, entre trois courbes expérimentales relatives aux mesures des efforts obtenus dans les différentes zones. Les mesures obtenues dans le cas du modèle à parois rugueuses et leur comparaison sont présentées dans la figure 20. 


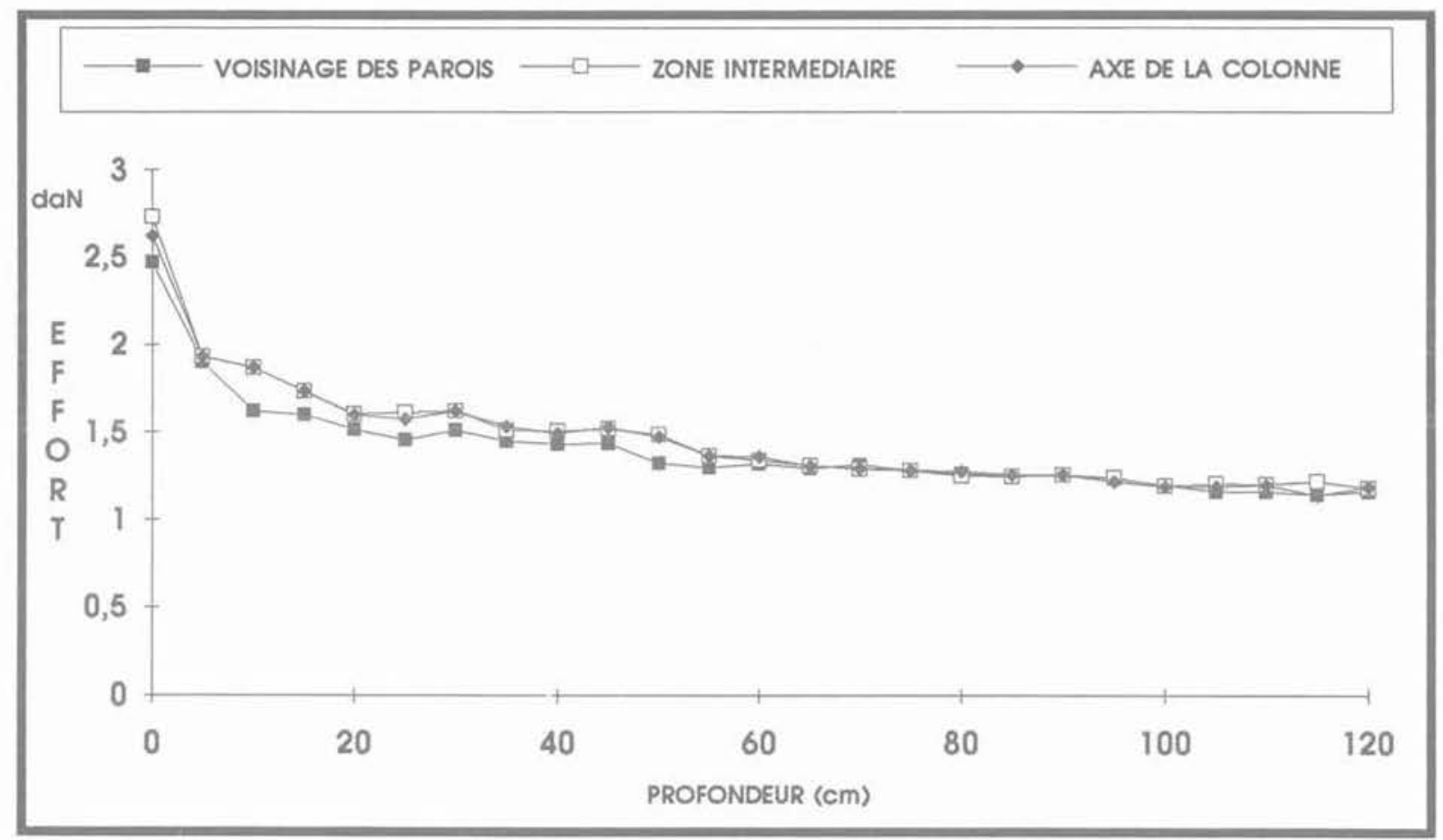

Fig. 19. - Influence de l'état de surface (parois semi-rugueuses).

Fig. 19. - Influence of the surface state lin the case of semi-wrinkled walls).

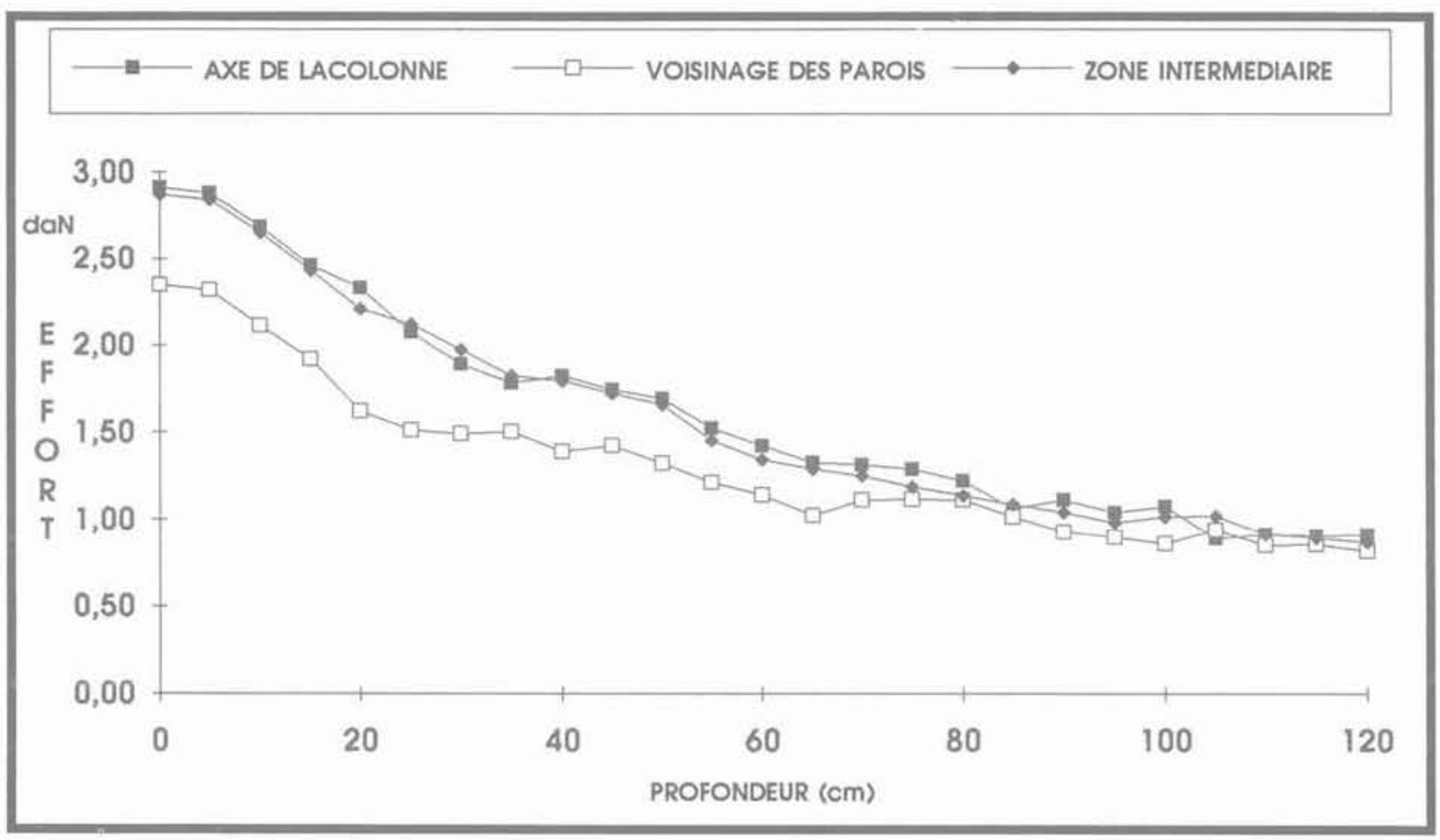

Fig. 20. - Influence de l'état de surface (parois rugueuses).

Fig 20 - Influence of the surface state lin the case of wrinkled wallst.

Analyse :

Tout d'abord, il est intéressant de constater que, sur ces figures, la contrainte isotrope au voisinage des parois est, dans $80 \%$ des cas, plus faible que celle au centre du massif. On peut constater aussi que les valeurs finales (les valeurs qui correspondent au fond du modèle) sont sensiblement identiques, que ce soit dans le cas des parois rugueuses ou dans le cas des parois semi-rugueuses, puisque dans cette partie, les courbes sont confondues jusqu'à une certaine côte $Z$ d'environ $80 \mathrm{~cm}$. Au-delà de cette hauteur, on cons- 
tate que les valeurs mesurées sont différentes, sauf dans le cas des parois semi-rugueuses où cette différence n'est pas trop élevée.

D'un point de vue expérimental, cela signifie que les effets de paroi sont négligeables dans le cas où cellesci sont lisses. En fait, la localisation du domaine influencé par la rugosité présente un grand intérêt du point de vue expérimental. Il serait donc nécessaire d'apporter certaines précisions supplémentaires dans ce domaine et d'expliquer en particulier les raisons pour lesquelles cette rugosité influe sur la répartition des contraintes au sein d'un massif granulaire.

On peut donc dire que linfluence de la rugosité sur la répartition des contraintes augmente très vite lorsqu'on se rapproche de la paroi et surtout quand celle-ci est rugueuse. En fait, l'influence minimale n'est pas obtenue dans la zone intermédiaire mais dans laxe de la colonne. Cependant toutes les courbes montrent que cette influence est atténuée à partir d'une profondeur $Z$ de l'ordre d'environ $60 \mathrm{~cm}$, et au fur et à mesure que la profondeur augmente, cette influence diminue et tend à disparaître à la base. Le calcul des coefficients de variation confirme cette analyse dans la mesure où ceux-ci sont presque constants dans les trois zones du modèle à parois semirugueuses. En revanche, au fur et à mesure que la rugosité des parois augmente, on constate une différence notable entre les coefficients de variation dans les différentes zones, mais ceux-ci sont plus importants au voisinage des parois que dans les deux autres zones. En conséquence, on peut confirmer que la rugosité a une très grande influence au voisinage des parois sur la répartition des contraintes et, de ce fait. peut modifier l'équilibre de la masse ensilée.

\section{CONCLUSION}

On vient de voir qu'à laide de la technique expérimentale de mesure des efforts nécessaires pour déplacer un ensemble de rouleau, il était possible de décrire la répartition des contraintes au sein du matériau. Ainsi, il a été confirmé que dans un silo contenant de la matière, soumis à une charge ou non, les efforts n'augmentent pas indéfiniment lorsque la hauteur de matériau croît; ils tendent vers des limites, comme diverses lois de détermination des efforts statiques dans les silos le montrent. Les résultats obtenus coïncident notamment avec ceux de JANSSEN. Les théories plus récentes n'ont pas fourni de meilleurs résultats compte tenu, notamment de la forte variabilité des caractéristiques mécaniques des milieux granulaires.

Durant la vidange, le matériau situé directement audessus de l'orifice forme une cheminée d'écoulement. la paroi sert de limite à celle-ci. L'examen détaillé des déplacements et des vitesses a permis de proposer différents modes de comportement du matériau au sein du modèle au cours de l'écoulement et ceci suivant la rugosité des parois et l'inclinaison de la trémie.
On a ainsi pu signaler l'existence d'une zone de cisaillement au niveau des parois. A ce propos, il est intéressant de souligner que le modèle utilisé permet des observations irréalisables sur des silos prototypes et que les prises de vue vidéo peuvent être utilisées pour établir les trajectoires des particules au sein du matériau ensilé.

En ce qui concerne le frottement entre le milieu et les parois, on a constaté, dans le cas des parois semirugueuses, que les effets de celles-ci sont négligeables. En revanche, dans le cas des parois rugueuses, le frottement entre elles et le matériau a une influence non négligeable au voisinage des parois : cela peut modifier l'équilibre de la masse ensilée. Naturellement, il faut rappeler que le matériau analogique de SCHNEEBELI est un modèle dans lequel les particules sont rondes et sans cohésion ; ce qui limite nécessairement l'existence des voûtes de charges.

\section{BIBLIOGRAPHIE}

(1) ABRIAK N. (1991), Ecoulement d'un matériau granulaire à travers un orifice : effet de paroi. Thès de Doctorat de l'Université des sciences et techniques de Lille, Flandres, Artois,

(2) CALIL C.J. (1988), Experimental study of compact loads in silos with funnel flow. SilosForschung und Praxis Tagung'88, Universität Karlsruhe.

(3) EIBL J., ROMBACH G. (1987), Stress and velocity fields at discharging of silos. Proc. of numeta. Swansea July.

(4) FAUGERAS J.C., GOURVES G. (1980), Mesure des contraintes au sein d'un massif analogique de SCHNEEBELI. Revue Française de Géotechnique $n^{\circ} 11$

(5) GOURVES R., MEZGHANI F. (1988), Micromécanique des milieux granulaires. Approche expérimentale utilisant le modèle de SCHNEEBELI. Revue Française Géotechnique n ${ }^{\circ} 42$.

(6) HATAHURA Y., TAKENCHI T., NAGAO T. (1988). Similitude of stress in silo (from small model to large on Scale Modelling. Tokyo, Japan, p. 277-278

(7) JANSSEN M.A. (1895), Versuche übergetreichedruck in silozellen. Zeifschrift verein deutscher Ingenieure, Vol. 39, p. 1045-1049.

(8) KHELIL A., ROTH J.C. (1989), Theoretical and experimental studies of stresses in full scale silos at the chartres experimental base. Powder and grains, BIAREZ et GOURVES.

(9) KHELIL A., ROTH J.C. (1990), Spécification des charges et des écoulements dans les silos métalliques. Revue Française de Géotechnique $n^{\circ} 52$

(10) TEJCHMAN J. (1988), A novel approach to the emptying mechanism of silos. Silos-Forzschung und Paradix Tagung'88. Universität Karlsruhe. 